En somme, la théorie que M. Alliévi a donnée des coups de bélier dans les conduites forcées, se trouve en grande partie vérifiée par les expériences d'Allevard et l'on peut dès lors se baser sur cette théorie el sur les formules auxquelles elle aboutit pour calculer les coups de bélier dans les différents cas de la pratique. Toutefois, la théorie n'est bien vérificée que pour la première onde. Les ondes ultérieures, modifiées sans doute par la viscosité de l'eau et par le frottement le long des parois ou encore par l'intertérence des ondes directes et réfléchies, se déforment el tendent rapidement à devenir sinusoïdales malgré que, si la fermeture a été brusquc, la première onde soit de forme trapézoïdale.

La durée de la période n'est pas exactement égale à $\frac{4 \mathrm{~L}}{a}$; elle scmble être généralement un peu plus longue.

Cahier des charges. - L'étude de ce dernier point de votre programme ne pouvait etre abordéc que lorsque toutes les autres questions auraient trouvé Jemr solution. Elle n'a par conséquent pu être entreprise.

Questions diverses. - Diverses questions ont été traitées dans nos réunions ; des difficultés inattendues surgissaient quand il s'agissait d'établir certains organes appelés à fonctionner avec exactitude dans des conditions de charge qui ne sont pas encore prévues par les constructeurs ; des apparils nouveaux ont été soumis à notre examen par leurs inventeurs.

La Revue La Houille Blanche, constamment mise avec la plus grande obligeance à la disposition de la Commission, a reproduit plusieurs des notes qui nous avaient été présentécs. L'index bibliographique joint à ces pages en fourjit la liste. (Il sera donné dans le prorhain numéro.)

Nous ne pouvons ici signaler les autres que pour mémoire.

Tel qu'il est, cet exposé prouve que votre Commission a travaillé : vous pouvez vous rendre témoignage que votre dévouement et vos peines n’ont pas été dépensés en vain. Les minules de tous vos travaiux se retrouveront dans vos archives, que nous vous proposons de remettre à la Société Hydrotechnique de France.

La Chambre Syndicale a bien voulu gérer les fonds qui nous avaient été remis et qu'clle a considérablement augmentés : elle est propriétaire d'une certaine partie de l'outillage dont nous avons usé ; l'autre apparlient à l'Association Lyonnaise des Propriétaires d'appareils ì vapeur visà-vis de laquelle la Chambre Syndicale en reste responsable.

Notre œuvre est donc terminéc, du moins en apparence, car elle sera poursuivie sous une forme différente; mais avec le même but, celui que dès la première heure vous vous ètes proposé en répondant à l'appel de Charles PrNat : servir les intérêts de l'industrie hydraulique et par delà ces intérêts zeux mêmes de la Patrie française.

\section{JAUGEAGES PAR DÉVERSOIRS ÉPAIS}

L'Engineering Record du r6 février $\mathrm{x} 913$, public un résưmé d'une note que le professeur SACKETT a présentée à l'Indiana Engineering Society, et où il a exposé les résultats des expériences de jaugeages par déversoirs épais, que MM. KEMMERer et LANE ont faites au Laboratoire d'Hydraulique de l'Université de Purdue, pour l'obtention du grade de docteur-ingénieur.

Les déversoirs expérimentés, au nombre de quatre, étaient itablis au travers d'un canal de roo pieds de long sur
3 pieds de large, dont ils occupaient toute la largeur. Deux de ces déversoirs étaient de section rectangulaire, tandis que les deux autres étaient à profil courbe. Les débits étaient mesurés au moyen d'un compteur Venturi, et les hauteurs étaient appréciées par le déplacement d'une tige terminée par un crochet.

Le déversoir $n^{0} \mathrm{I}$, à section restangulaire, avait 0,98 piedș de large sur 2,38 pieds de haut. Le déversoir $\mathrm{n}^{\circ} 2$ avait 0,48 pied de large sur 2,27 pieds de haut. Pour ces deux déversoirs, des précautions ont été prises pour admettre l'air sous la lame déversoir, de façon à y éviter la formation du vide.

Le profil du déversoir $n^{0} 3$ se composait d'un arc de cercle, de un pied de rayon, se prolongeant a l'amont et a l'aval suivant les tangentes à $145^{\circ}$. La hautcur de ce déversoir ćtait de 2,5 pieds:

Le profil du déversoir $\mathrm{n}^{\circ} / 4$ comportait également un arc de cercle de un pied de rayon, mais si le parement aval était encore constitué par la tangente à $45^{\circ}$, le parement amont était vertical, ol situé à 7 pouces du centre de l'are de cercle.

\begin{tabular}{|c|c|c|c|c|c|}
\hline 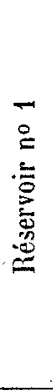 & $\begin{array}{c}\mathrm{H} \\
0,211 \\
0,264 \\
0,331 \\
0,385 \\
0,435 \\
0,447 \\
0,497 \\
0,548\end{array}$ & $\begin{array}{c}0 \\
0,263 \\
0,359 \\
0,503 \\
0,635 \\
0,761 \\
0,787 \\
0,937 \\
1,090\end{array}$ & $\begin{array}{c}\mathrm{C} \\
2,71 \\
2,64 \\
2,61 \\
2,66 \\
2,65 \\
2,63 \\
2,68 \\
2,69\end{array}$ & $\begin{array}{l}\text { 量 } \\
\underline{\Xi} \\
\vdots \\
0 \\
0 \\
11 \\
0\end{array}$ & $\begin{array}{c}l \\
-1,5 \\
+0,8 \\
-1,0 \\
-0,5 \\
-0,7 \\
+1,4 \\
-0,1 \\
-0,6\end{array}$ \\
\hline 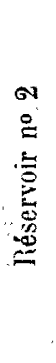 & $\begin{array}{l}0,212 \\
0,261 \\
0,313 \\
0,367 \\
0,368 \\
0,424 \\
0,462 \\
0,518 \\
0,568\end{array}$ & $\begin{array}{l}0,250 \\
0,347 \\
0,463 \\
0: 613 \\
0,604 \\
0,780 \\
0,933 \\
1,130 \\
1,330\end{array}$ & $\begin{array}{l}2,56 \\
2,60 \\
2,65 \\
2,76 \\
2,71 \\
2,82 \\
2,97 \\
3,03 \\
3,10\end{array}$ & 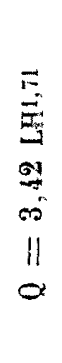 & $\begin{array}{l}-3,04 \\
-0,6 \\
+1,7 \\
+0,8 \\
+2,6 \\
+1,4 \\
-2,0 \\
-1,6 \\
-2,3\end{array}$ \\
\hline 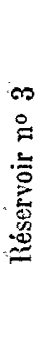 & $\begin{array}{l}0,074 \\
0,114 \\
0,177 \\
0,238 \\
0,297 \\
0,341 \\
0,415 \\
0,474 \\
0,539\end{array}$ & $\begin{array}{l}0,060 \\
0,112 \\
0,223 \\
0,359 \\
0,516 \\
0,+56 \\
0,903 \\
1,140 \\
1,100\end{array}$ & $\begin{array}{l}3,01 \\
2,91 \\
2,98 \\
3,09 \\
3,18 \\
3,30 \\
3,39 \\
3,50 \\
3,54\end{array}$ & 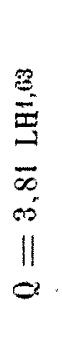 & $\begin{array}{r}-1,4 \\
-1,8 \\
+0,9 \\
+1,5 \\
+1,7 \\
+0,2 \\
+0,2 \\
-1,8 \\
-0,7\end{array}$ \\
\hline 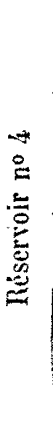 & $\begin{array}{l}0,096 \\
0,168 \\
0,209 \\
0,246 \\
0,305 \\
0,339 \\
0,364 \\
0,383 \\
0,448 \\
0,452 \\
0,557\end{array}$ & $\begin{array}{l}0,081 \\
0,207 \\
0,293 \\
0,388 \\
0,588 \\
0,634 \\
0.711 \\
0,785 \\
1,016 \\
1,007 \\
1,390\end{array}$ & $\begin{array}{l}2,82 \\
3,01 \\
3,07 \\
3,18 \\
3,20 \\
3,22 \\
3,23 \\
3,24 \\
3,38 \\
3,31 \\
3,34\end{array}$ & 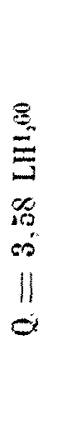 & $\begin{array}{r}0,7 \\
0,0 \\
0,0 \\
-2,1 \\
-0,3 \\
+0,2 \\
+0,1 \\
+0,4 \\
-2,3 \\
-0,1 \\
+1,4\end{array}$ \\
\hline
\end{tabular}

Le tableau ci-dessus donne les résultats obtenus au cours de quélques-unes de ces expériences. On en a déduit les valeurs de $\mathrm{C}$ de la formule : $\mathrm{Q}=\mathrm{CL} \mathrm{H} \mathrm{H}^{3 / 2}$, ainsi que les coefficients d'une formule exponentielle concordant avec 
la moyenne des résultats acquis sur un même modèle ${ }^{(1)}$.

La dernière colonne: du tableau indique la différence $d$, en pour cent, des résultats obtenus par l'emploi des types de formules.

Tous les chiffres de ce tableau sont exprimés en pieds. Le débit $Q$ se rapporte à une largeur de déversoir de un piod, et la hauteur $H$ indiquée représente la charge sur le déversoir augmentée de la charge correspondante à la vítesse d'arrivée de l'eau sur le déversoir.

Si l'on trace la courbe représentalive des variations du coefficient $\mathrm{C}$, on remarque que cette courbe s'écarte peu d'une droite pour le déversoir $n^{0} 3$, tandis qu'elle présente une forme courbo très accusée par le déversoir $n^{\circ} 4$. Pour les très faibles charges, l'influence idu bec amont du dévorsoir $n^{0} 3$ par rapport au $n^{\circ} 4$ est peu sensible, et, on tous cas, paraîtrait favoriser l'écoulement; par contre, pour les charges au-dessus de 3 pieds, olle a pour elfot de diminuer notablement la valeur du coefficient $\mathrm{C}$, et par suite cello dù débit.

\section{LA FABRICATION DU FERR0-MANGANESE AU FOUR ÉLECTRIQUE} -ísuite ET FIN ) -

Dans le numéro précédent de La Houille Blanche, nous avons opposé le four électrique au haut fourneau pour la fabrication des iferros-manganèse. Nous voulons aujourd'hui parler des matières premières employées dans cette industrie el des usages, de jour en jour plus nombreux, de ce produit.

\section{MATIERES PREMIERES}

Minerais de manganèse. - Ils se rangent au point de vue minéralogique en deux groupes : les mincrais oxydés et hydratés d'uno part, et les minerais carbonatés et silicatés de l'aulre.

Tes minerais oxydés les plus abondants sont : la psilomélane, la polianite, la pyrolusite. La manganite, la braunite, Io haussmannite et le wad se trouvent en plus petite quantilé. - Les minerais carbonalẹ́s et silicatés, le manganèse spathique et les minerais silicieux ont une teneur plus faible en manganèse que les minerais oxydés.

On trouve les minerais de manganèse en affleurement, couches de profondeur ou métamorphisés. Ces groupes de gîtes ont beaucoup de points communs avec les couches ferrugineuses et forment avec ces dernières les groupes définis de minerais ferro-manganésifères.

Les minerais à forte teneur sont extraits principalement de la Russie; du Brésil et des Indes ; on en trouve aussi de petites quantités en Turquie, en Grèce, en France el en Espagne.

La Russie est le pays le plus riche en minerais de manganèse ; les $8 / 9$ environ de sa production provicnnent du Caucase, on en extrait aussi à Iékaterinoslaw el une pelite quantité dans l'Oural.

Dans le Ciancase, le gisement le plus important est cclui de Tschiaturi, sur le Kivirila. La région manganésifère arrosée par le Kivirila est un plateau bas partagé par de nombreuses vallées ; le sol est formé par des terrains tertiaires et crayeux, qui. reposent en profondeur sur des couches graphitiques et syénitiques. Ce minerai est dirigé sur Poti

(1) Ces coefficients ne seraient plus les mêmes avec l'application du syoteme métrque Rappelons que le pied vaut om 30470 . et surtout sur Batoum, qui est le grand port d'expédition du minerai de manganèse pour l'Allemagne.

Au Brésil, les gisements de minerai de manganèse lés plus importants sont, d’après Kausch, ceux de la Miguel, Burnier Mine, dans le district de Minas Geraes ; de Lafayette ou Queluz, dans le voisinage de l'Ouro-Preto ; de Nazareth, dans le voisinage de San Salvador (district de Bahia) ; de Matto Grosso, au sud de Korumba ; de Macuara, dans l'Amazone, etc.

Le district brésilien le plus important au point de vue mincrai de manganèse est le Miguel Burnicr, sur le Chemin de Fer Central, près de 'l'Ouro-Preto.

Le sous-sol du pays est formé d'une couche de schistes ardoisiers d'une puissance de ro mètres ; on trouve all-dessus des calcaires dolomiliques à environ $x, 50 \%$ de manganèse et, enfin, une couche de minerai de mangasèse, dont la teneur alteint $20 \%$ pour cent.

D'après Scott, il y a là deux millions de tonnes de minerai exploitables. Ce minerai convient particulièrement bien poür la fabrication du ferro-manganèse pauvre en phosphore.

Aux Indes, la plus grande partie des minerais de manganèse provient des provinces centrales $\mathrm{d}$, en particulier, des districts de Vagpur, Balaghat, Bhandara, Chindivara et Vizagapatam, etc...

En Turquie, le gisement de Kassandra est le plus importánt. En Grèce, c'est le district minier de Milos. Le minerai de Milos est peu estimé, car il est très riche en oxyde do manganèse (Mno) silicaté, dont la réduclion est difficile. I.es mincrais ù faible teneur de Mn sont rares en Grèce.

La France livre des oxydes de manganèses combinés avec du carbonate et du silicate. Jc ne ferai mention que du minerai calcinć de Las Cabasses, dans le département de 1'Ariège et de celui de Romanèche.

Le domaine minier entre la Garonne et l'Ariège est composé de couches paléozoïques, iétroitement pressées, orientécs. de l'ouest à l'est. Le minerai est 'du carbonate pur, en rognons ou bạtonnets, transformé en oxyde aux affleurements.

Les fosses de Las Cabasses sont situées dans la vallée de la Nert et appartiennient à la, Société "Las Cabasses Mine Ld".

Le minerai de Romanèche est un genre de psilomelane ;; sa composition est si constante que M. Lacroix, membre de l'Institut, professeur au Muséum, en a fait une espèce distincte qu'il appelle la Romanéchite, dont la formule serait.: $\mathrm{H}^{2}(\mathrm{Mn} \mathrm{Ba}) \mathrm{Mn}^{3} \mathrm{O}^{8}$.

M. Gorgen écrit cetle formule : $(\mathrm{MnBa}) \mathrm{O}, 3 \mathrm{MnO}^{2}+\mathrm{H}^{2} \mathrm{O}$. - L'eau paraît combinée.

Voici l'analyse de la Romanéchite donnée par M. Chamussy, directeur des mincs de Romanèche : $\mathrm{MnO}^{2}, 63,27 \%$; $\mathrm{Fe}^{2} \mathrm{O}^{3}, 3,50 \% ; \mathrm{SO}^{4} \mathrm{Ca}+\mathrm{Al}^{2} \mathrm{O}^{3}+\mathrm{SiO}^{2}, \mathrm{r} 3,30 \% ; \mathrm{BaO}+$ $\mathrm{CaO}+\mathrm{MgO}, \mathrm{r} 6,47 \% ; \mathrm{CO}^{2}$, corps non dosés, $3,46 \%$. Soit au total $39,94 \%$ de mangánèse métal.

La Romanéchite forme des masses compactes ou concrélionnéeś à texture fibreuse; les surfaces libres des géodes sont le plus souvent mamelonnées, stalacliformes, botrogides ; elles sont quelquefois recouvertes d'aiguilles fines donnant à la surface un aspect velouté ; il existe des qualités pulvérulentes ; la coulcur du minẹrai est bleu d'acier ou noir ; certains échantillons présentent une cassure irisẹ́e de dịverses couleurs (queuc de paon).

Les minerais de manganèse de Romanèche étant trè̀s denses et très durs, se prêtent admirablement au traitement métallurgique ct surtout électrothermique, c'est pourquoi nous avons cru devoir leur réserver une place dáns cette étude: Son prix varie de $0,7^{5}$ ì 1,10 l'unite de manganèses. 